\title{
Autophagie et vision
}

\section{Beatriz Villarejo-Zori, Patricia Boya \\ Traduction française de Thierry Jouault}

> La rétine est un tissu de l'œil des vertébrés sensible à la lumière qui détecte et traite les images visuelles. L'œil et la rétine sont soumis à une variété d'agressions et de stress environnementaux, des modifications liées à l'âge et des mutations génétiques qui nuisent à leur fonction. L'autophagie est une voie catabolique qui permet la dégradation et le recyclage des composants cellulaires, en conditions physiologique ou de stress. Des protéines, des lipides, et même des organites entiers sont ainsipris en charge par les autophagosomes et transportés dans les lysosomes afin de les éliminer. Dans le système visuel, le rôle de l'autophagie commence à être étudié. Nous relaterons dans cette revue les principales études montrant l'importance de l'autophagie dans la fonction visuelle. <

La rétine est un tissu sensible à la lumière qui détecte et traite les images visuelles. Doublant la surface interne de l'œil, elle détecte la lumière et crée des impulsions qui transitent par le nerf optique jusqu'au cerveau. Décrite par Santiago Ramón y Cajal, il y a plus de 100 ans, la rétine est composée de plusieurs types de cellules disposées en couches (Figure 1): trois couches de corps de cellules nerveuses et deux couches de synapses. La couche nucléaire externe (Figure 1A) est constituée des photorécepteurs, des cellules sensibles à la lumière regroupées en deux grandes classes, les bâtonnets et les cônes (Figure IA, B). Ces cellules se connectent au niveau de la couche plexiforme externe (Figure 1C) avec des cellules amacrines et bipolaires dont les noyaux forment la couche nucléaire interne (Figure 1D). Dans la couche plexiforme interne (Figure $1 \varepsilon$ ), les cellules amacrines bipolaires se connectent avec les cellules rétiniennes ganglionnaires (RGC) (Figure IF), les seuls neurones de projection de la rétine dont les axones forment le nerf optique (Figure 1G) qui la relie au cerveau. À côté

Vignette (Photo @ Inserm-Hanon Khabou).

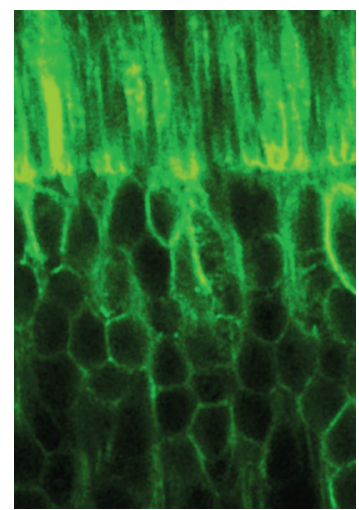

de ces cellules nerveuses, la rétine est également constituée de cellules gliales, appelées cellules de Müller. Localisées immédiatement à l'extérieur de la neurorétine, en contact étroit avec les cellules photoréceptrices, les cellules épithéliales rétiniennes pigmentées (RPE) participent au renouvellement du segment extérieur des photorécepteurs, tout en leur fournissant un support.

L'autophagie est une voie catabolique permettant la dégradation et le recyclage des composants cellulaires. Des protéines, des lipides et des organites entiers (mitochondries) sont ainsi pris en charge par des autophagosomes et délivrés aux lysosomes afin de les éliminer

$\rightarrow$ Voir la Nouvelle de $\varepsilon$. Morel, page 217 de ce numéro [47] $(\rightarrow)$.

En réponse au stress, ce processus permet la dégradation de composants cellulaires altérés qui sont recyclés et permettent l'apport de nutriments nécessaires au maintien de l'homéostasie cellulaire. L'autophagie joue un rôle important dans le contrôle de qualité cellulaire, en particulier pour les neurones, dans lesquels les protéines altérées et les organelles endommagées ne peuvent être redistribuées, après division cellulaire, à des cellules filles.

Les recherches sur le rôle de l'autophagie dans le système visuel ne sont qu'à leurs prémices. La rétine, et l'œil en général, sont exposés à une grande variété d'agressions et de facteurs de stress environnementaux. Ils sont également sujets à diverses mutations génétiques et soumis aux modifications liées à l'âge qui altèrent leur fonction [1]. Nous présentons, dans cette revue, les principales études portant sur l'importance de l'autophagie dans la fonction visuelle. En examinant les conséquences de la régulation de l'autophagie dans différents types de cellules rétiniennes, nous aborderons le rôle essentiel des régulateurs de ce processus dans le maintien de I'homéostasie de la rétine dans des conditions physiologiques et dans des situations de stress. Nous décrirons également le rôle que 


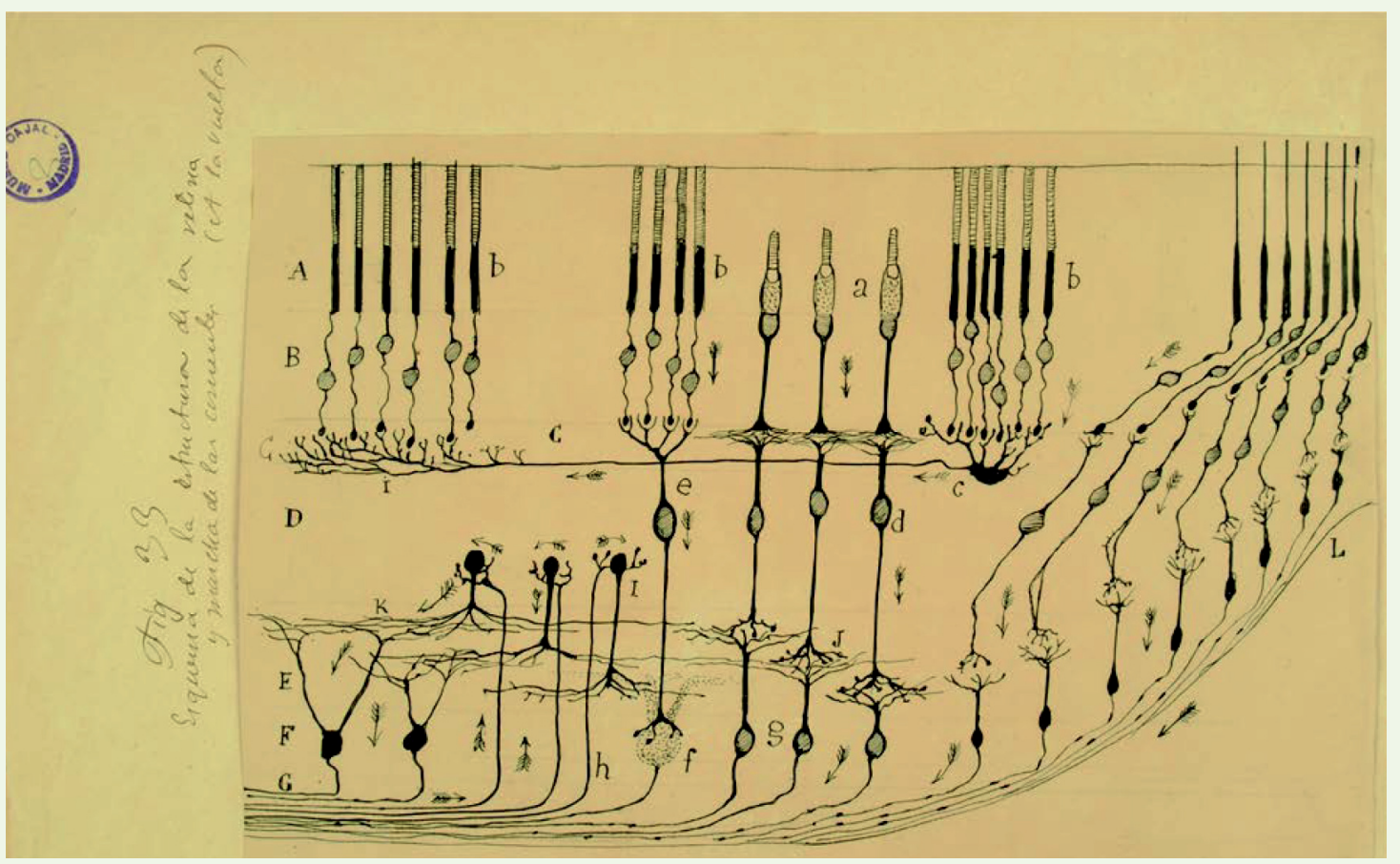

Figure 1. Représentation schématique de la structure de la rétine de mammifère telle que décrite par Santiago Ramón y Cajal (1901). A. Couche de cônes et de bâtonnets. B. Couche nucléaire externe. C. Couche plexiforme externe. $\boldsymbol{D}$. Couche de cellules bipolaires. $\boldsymbol{\varepsilon}$. Couche plexiforme interne. F. Couche de cellules ganglionnaires. G. Nerf optique. L. Fosse centrale (reproduit du dessin original avec la permission du Cajal Institute, CSIC, Madrid, Espagne).

joue l'autophagie dans la survie des photorécepteurs et dans des pathologies liées à l'âge, comme la dégénérescence maculaire et le glaucome. Nous discuterons enfin de la possibilité de l'utilisation de l'autophagie pour la protection des cellules de la rétine avec, pour corollaire, l'évocation de nouvelles approches thérapeutiques permettant de remédier à la cécité et à d'autres maladies de l'œil.

\section{Rôle de l'autophagie dans les cellules rétiniennes : des preuves issues des analyses d'animaux déficients pour l'autophagie}

Depuis la découverte des gènes ATG (autophagy related genes), de nombreux modèles animaux déficients ont été générés permettant de comprendre le rôle de l'autophagie dans différents tissus. En général, l'absence totale d'autophagie se traduit par une mort embryonnaire ou postnatale précoce [2]. L'incidence des déficiences génétiques sur la rétine ne peut donc être évaluée dans ces modèles «classiques 》. En revanche, les modèles de déficit partiel, comme les souris déficientes en $A T G 4 B$ qui survivent jusqu'à l'âge adulte, représentent un excellent modèle pour l'étude de l'autophagie dans les tissus à l'âge adulte. Les souris déficientes en ATG4B ne présentent pas de modifications majeures sur le plan histopathologique ou biochimique bien que l'autophagie soit réduite (mais pas abolie) dans de nombreux tissus
[3], y compris au niveau de la rétine [4]. En effet, dans des conditions physiologiques normales, les rétines de souris $A T G 4 B^{-1-}$ ne présentent pas d'altération. Elles sont cependant plus sensibles au stress, l'axotomie ${ }^{1}$ du nerf optique conduisant chez ces animaux à une survie réduite des cellules rétiniennes ganglionnaires (RGC) par rapport aux souris sauvages $[4,5]$. Ces données suggèrent donc que même légère, une réduction du niveau d'autophagie rétinienne peut altérer la capacité des cellules à répondre à un stress. Les souris $B E \mathrm{CNI}^{+/-}$(I'homologue d'ATG6, qui code la protéine autophagique Beclin-1) ne présentent pas, elles non plus, de modifications morphologiques de la rétine mais l'exposition à la lumière brillante de ces animaux provoque des lésions avec dégénérescence de la rétine et altérations des photorécepteurs [6]. Ces souris présentent également des atteintes des cellules épithéliales rétiniennes pigmentées (RPE) avec des dommages nucléaires, une vacuolisation cytoplasmique et des mitochondries enflées et altérées. Ces deux modèles animaux de déficience partielle d'autophagie indiquent

${ }^{1}$ Section d'un axone. 


\begin{tabular}{|c|c|c|c|c|}
\hline $\begin{array}{l}\text { Déficience de } \\
\text { l'autophagie }\end{array}$ & Modèles animaux & Altérations rétiniennes & Phénotypes & Références \\
\hline Rétine neurale & $\begin{array}{l}\text { ATG5 } 5^{f l o x} / f l o x \\
\text { Nes-Cre }\end{array}$ & $\begin{array}{l}\text { Mort des PR, gliose } \\
\text { Réduction de la fonction visuelle }\end{array}$ & Vieillissement & {$[8]$} \\
\hline RGC & $\begin{array}{l}\text { ATG5 flox/flox } \\
\text { AAV2-Cre-GFP } \\
\text { AAV2-GFP }\end{array}$ & $\begin{array}{l}\text { Mort des RGC après axotomie du nerf } \\
\text { optique }\end{array}$ & Glaucome & {$[4]$} \\
\hline RPE & $A T G 5^{\triangle R P E}$ & $\begin{array}{l}\text { Pas d'altération des PR jusqu'à } 6 \text { mois } \\
\text { Réduction des pigments visuels } \\
\text { Réduction de la fonction visuelle }\end{array}$ & Vieillissement & [9] \\
\hline RPE & $A T G 7^{\triangle R P E}$ & $\begin{array}{l}\text { Histologie normale à } 12 \text { mois } \\
\text { Pas d'altération du cycle visuel } \\
\text { Fonction visuelle normale }\end{array}$ & DMLA & {$[10]$} \\
\hline RPE & $\mathrm{RBICCl}^{\mathrm{CKO}}$ & $\begin{array}{l}\text { Dégénérescence des cellules RPE à } \\
8 \text { mois } \\
\text { Diminution de l'épaisseur de l'ONL }\end{array}$ & DMLA & [12] \\
\hline Cônes & ATG5 $5^{\Delta C o n e}$ & $\begin{array}{l}\text { ONL normal } \\
\text { Dégénérescence des cônes } \\
\text { à partir de } 4 \text { mois } \\
\text { Réduction de la fonction visuelle à } \\
8 \text { mois } \\
\text { Sensibilité au stress lumineux }\end{array}$ & $\begin{array}{l}\text { Dégénérescence des cônes } \\
\text { Dommages dus à la lumière }\end{array}$ & [14] \\
\hline Bâtonnets & $A T G 5^{\triangle R o d}$ & $\begin{array}{l}\text { Réduction de l'ONL à } 2 \text { mois } \\
\text { Mort des photorécepteurs } \\
\text { Insensibilité au stress lumineux }\end{array}$ & $\begin{array}{l}\text { Dégénérescence des bâtonnets } \\
\text { Dommages dus à la lumière }\end{array}$ & [13] \\
\hline Bâtonnets & ATG $7^{\Delta R o d}$ & $\begin{array}{l}\text { Pas d'anomalie } \\
\text { Sensibilité au stress lumineux }\end{array}$ & Dommages dus à la lumière & [6] \\
\hline
\end{tabular}

Tableau I. Principaux phénotypes rétiniens des modèles murins de déficience d'autophagie. PR : photorécepteurs ; RGC : retinal ganglion cell ; ONL : outer nuclear layer; DMLA : dégénérescence maculaire liée à l'âge ; RPE : cellules épithéliales rétiniennes pigmentées; ATG : autophagy-related genes ; AAV2 : adeno-associated virus 2 ; GFP : green fluorescent protein ; Cre : tyrosine recombinase issue du bactériophage Pl ; Nes : nestin ; RBICC1 : RB1[retinoblastoma-associated protein]-inducible coiled-coil 1.

ainsi qu'une réduction même légère des niveaux d'autophagie peut se traduire par des altérations de la rétine dans des conditions de stress. Des modèles de souris déficientes en autophagie spécifiquement au niveau de plusieurs types de cellules ont été générés. Les phénotypes obtenus au niveau rétinien sont résumés dans le Tableau I. Les premières souris ont été invalidées pour des gènes de l'autophagie spécifiquement dans les précurseurs des neurones. Dans ce cas, la progéniture présentait un phénotype neurodégénératif évident, entrầnant la mort des animaux à environ 3 mois [7]. Ces souris présentaient également des altérations rétiniennes importantes, dès 5 semaines de développement, avec une augmentation des niveaux de p62 (un partenaire de l'autophagie récepteur des protéines ubiquitinées), une accumulation d'ubiquitine dans toutes les couches de la rétine, et la mort des cellules photoréceptrices [8] (Tableau I). Ces observations suggéraient donc qu'un niveau basal d'autophagie dans les précurseurs neuronaux était nécessaire afin de maintenir la physiologie normale de la rétine et que la diminution des niveaux d'expression des gènes d'autophagie, en particulier ATG5, conduisait à une neurodégénérescence des photorécepteurs [8].

\section{Régulation de l'autophagie dans les cellules} épithéliales rétiniennes pigmentées

Les cellules épithéliales rétiniennes pigmentées (RPE) sont localisées en dehors de la couche de photorécepteurs de la rétine neurale. Elles forment une couche cellulaire unique assise sur une membrane basale. Les cellules RPE sont essentielles pour le bon fonctionnement des photo- 
récepteurs. Elles leur fournissent un soutien nutritionnel en participant à leur renouvellement (photoreceptor disk shedding) par phagocytose, et à la dégradation et au recyclage des segments externes des cellules photoréceptrices apicales (POS), ce qui permet le renouvellement des pigments visuels. Deux modèles de souris déficientes en ATG5 ou en ATG7 spécifiquement au niveau de l'épithélium pigmentaire de la rétine, ont été générés. Les jeunes animaux $A T G 5^{\triangle R P E}$ ont une fonction rétinienne normale. En revanche, les animaux plus âgés présentent une activité réduite des photorécepteurs qui, étonnamment, n'est pas corrélée à une réduction du nombre de cônes ou de bâtonnets, mais est le résultat d'une récupération insuffisante des rétinoïdes et de modifications ultérieures de la fonction visuelle [9]. Pour les souris déficientes en ATG7 spécifiquement dans les cellules RPE ( $A T G 7^{\triangle R P E}$ ), aucun effet n'a été identifié sur le recyclage des rétinoïdes ou la fonction visuelle chez les jeunes animaux [10]. Ces différences pourraient être expliquées par les souches de souris utilisées ou leur âge mais cela reste à confirmer (Tableau I). Des études récentes ont été menées sur des animaux invalidés pour le gène $R B I C C l$ (RBII[retinoblastoma-associated protein]-inducible coiled-coil 1) dans les cellules RPE. RBICCl, également connu sous le nom de FIP200 (FAK family kinase-interacting protein of $200 \mathrm{kDa}$ ), est l'un des composants du complexe formé entre ULKI (Unc-51-like autophagy activating kinase 1), ATG13 et FIP200, qui initie le processus d'autophagie, en aval de mTOR (mammalian target of rapamycin complex) [11]. Les souris dépourvues d'expression de RBICCI présentent une atrophie des cellules RPE dès l'âge de 4 mois, qui se traduit par des altérations marquées de la couche de cellules RPE avec une infiltration de macrophages et l'activation de la microglie [12]. L'accumulation, au niveau de ces cellules, de protéines et de lipides oxydés et une autofluorescence, suggèrent l'accumulation de lipofuscine (un pigment lipidique formé à partir des débris cellulaires), conséquence de la diminution d'autophagie. La perte d'autophagie chez ces animaux produit également un amincissement de la couche de photorécepteurs et, consécutivement, une altération de la fonction visuelle. Ces caractéristiques sont similaires aux dommages spécifiques observés dans la dégénérescence maculaire liée à l'âge (DMLA), ce qui suggère que l'activité de FIP200 dans les cellules RPE est essentielle pour le maintien de la fonction visuelle et que des perturbations de cette protéine conduisent à une altération progressive ressemblant à la DMLA [12]. L'autophagie a donc un rôle essentiel dans le maintien de l'homéostasie des cellules RPE et de la couche de cellules photoréceptrices nécessaires à la fonction visuelle.

\section{Des modèles de souris de déficience d'autophagie dans les photorécepteurs}

Les photorécepteurs sont des cellules nerveuses de la rétine qui capturent la lumière et déclenchent une chaîne de processus biologiques connus sous le nom de phototransduction. Les photorécepteurs rétiniens sont des cellules très spécialisées. II en existe de deux types, les bâtonnets et les cônes, qui sont respectivement responsables de la vision nocturne et de la vision diurne des couleurs. Bien que plusieurs mécanismes cytoprotecteurs existent, de nombreuses situations pathologiques provoquent l'altération et la mort de ces cellules, conduisant à des défauts de la vision. Une étude récente a évalué les conséquences de la suppression d'ATG7 dans les bâtonnets, qui permettent la vision en lumière faible [6] (Tableau I). Chez ces souris ATG ${ }^{\Delta R o d}$, aucune altération n'a été observée lorsque les animaux étaient en conditions d'éclairage normal. Cependant, comme dans le cas des souris $B E C N I^{+/-}$, l'exposition de ces souris à un éclairage intense induit une dégénérescence de la rétine et une mort des photorécepteurs [6]. La suppression d'ATG5 dans les bâtonnets se traduit par des résultats radicalement différents [13]. En effet, les souris $A T G 5^{\Delta R o d}$ présentent une perte des photorécepteurs, en relation avec leur mort apoptotique, qui dépend de l'âge. Cette dégénérescence qui est observée également chez des animaux qui ont été élevés dans l'obscurité, est induite par la lumière intense [13]. Chez ces souris $A T G 5^{\Delta R o d}$, une légère diminution du nombre de cônes est également observée, ce qui suggère que la suppression du gène ATG5 dans les bâtonnets peut également affecter les cônes. Dans les bâtonnets, la suppression des gènes ATG5 et ATG7 a donc des effets qui diffèrent mais, là encore, les conditions expérimentales pourraient expliquer ces divergences. Un modèle de souris dans lesquelles ATG5 a été supprimé spécifiquement dans les cônes a également été développé [14]. Les souris $A T G 5^{\Delta C o n e}$ présentent des altérations du nombre de cônes qui apparaissent très tôt avec l'âge, avec une fonction réduite des cellules à l'âge de 10 mois. Une accumulation de $\mathrm{p} 62$ dans les mitochondries et dans le segment intérieur des photorécepteurs est observée dans les cônes des souris $A T G 5^{\Delta \text { Cone }}$ âgées de 7 semaines, par rapport aux animaux sauvages. Ces amas dépendent de l'âge des animaux indiquant une progression de la dégénérescence des cônes photorécepteurs après modulation de l'autophagie. L'extinction spécifique d'ATG5 dans les cônes provoque, en outre, une sensibilité accrue à la lumière [14]. La suppression d'ATG5 dans les cônes rend ainsi ces cellules sensibles au vieillissement et à la dégénérescence induite par la lumière. L'autophagie semble donc essentielle pour préserver la survie des cônes et des bâtonnets, une déficience d'autophagie dans ces cellules les rendant plus sensibles aux dommages induits par des conditions de stress comme ceux provoqués par la lumière.

\section{Altération de l'autophagie dans les cellules ganglionnaires de la rétine}

Les cellules ganglionnaires rétiniennes (RGC) sont des neurones de la rétine qui font saillie; leurs axones forment le nerf optique, permettant la transmission des informations visuelles au cerveau [15]. Aucune souris présentant un déficit spécifique de l'autophagie au niveau des cellules ganglionnaires rétiniennes n'a 


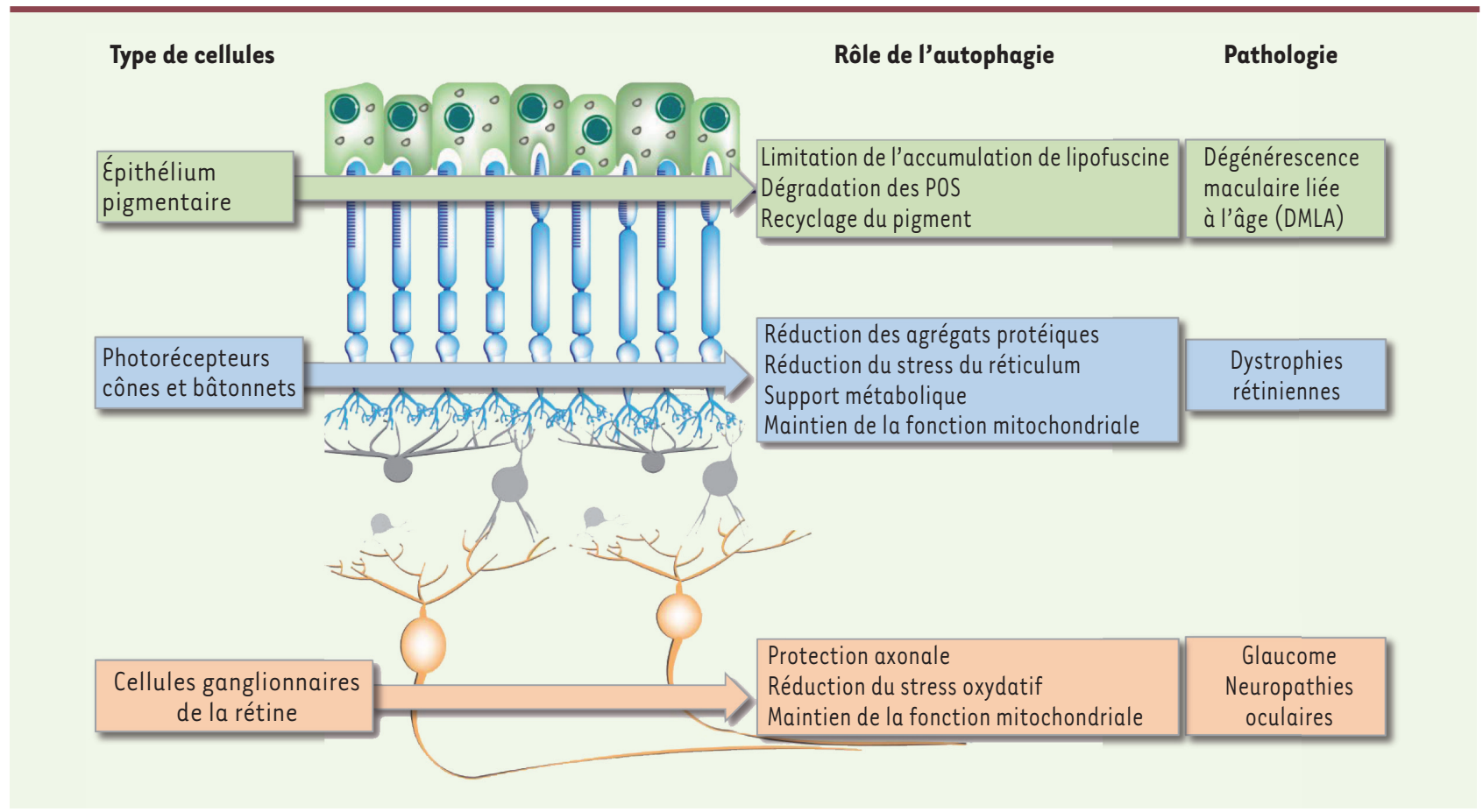

Figure 2. Rôle de l'autophagie dans les cellules rétiniennes et en condition pathologique en lien avec des altérations du processus d'autophagie. POS : segments externes des cellules photoréceptrices apicales.

été générée à ce jour. Néanmoins, des études fondées sur l'utilisation de vecteurs viraux comme AAV2 (adeno-associated virus 2) ciblant spécifiquement les RGC ont permis d'étudier les effets de la baisse de niveaux de l'autophagie dans ces cellules [4]. L'altération d'ATG5 rend ainsi les RGC plus sensibles à l'axotomie du nerf optique. Les souris ATG5 flox/flox, ayant reçu le vecteur AAV2-GFP-Cre, présentent en effet une réduction de la survie des cellules après axotomie [5], ce qui montre le rôle cytoprotecteur de l'autophagie pour la survie des RGC dans des conditions de lésions axonales.

Les processus d'autophagie apparaissent donc nécessaires pour préserver l'homéostasie de tous les types de cellules rétiniennes: la suppression d'ATG5, par exemple, étant à l'origine de phénotypes qui se rapprochent de ceux observés dans les maladies rétiniennes comme la DMLA. De nombreux modèles murins déficients en autophagie présentent une sensibilité accrue aux dommages induits par la lumière ou aux altérations axonales, soulignant ainsi l'importance de l'autophagie au cours de la réponse au stress cellulaire, dans la rétine.

\section{L'autophagie et les maladies rétiniennes}

\section{La dégénérescence maculaire liée à l’âge}

La dégénérescence maculaire liée à l'âge (DMLA ou, en anglais, AMD pour age-related macular degeneration) est une rétinopathie bilatérale affectant la macula, la région centrale de la rétine. Elle est une cause majeure de déficience visuelle chez les personnes âgées. La macula est le principal site de la vision photopique (ou diurne). Elle est constituée de la fovéa, une zone où se concentrent les cônes responsables de la vision diurne. La dégénérescence des cellules RPE est l'une des caractéristiques de la DMLA. Comme nous l'avons vu, les cellules RPE sont essentielles pour la survie et le bon fonctionnement des photorécepteurs. Elles apportent aux cellules les éléments nutritifs et sont responsables du renouvellement par phagocytose des cellules photoréceptrices apicales. Les cellules RPE sont des cellules post-mitotiques, ce qui suggère qu'elles présentent des niveaux élevés d'autophagie et autres mécanismes de détoxication. Ces cellules, résistantes au stress oxydatif, présentent une autophagie très active, ce qui améliore leur capacité à éliminer des structures cellulaires endommagées [16]. Elles sont exposées à des niveaux élevés d'oxygène et elles métabolisent une quantité importante de membranes cellulaires provenant des photorécepteurs exposés à une lumière constante. Ces conditions favorisent la formation de lipofuscine, qui entraîne des dommages cellulaires, dont des composants, comme le A2- $\varepsilon^{2}$, inhibent la pompe à protons lysosomale, l'augmentation de $\mathrm{pH}$ et l'activité des hydrolases lysosomales [17]. L'incubation in vitro de cellules RPE humaines immortalisées avec des segments externes de photorécepteurs induit l'accumulation de lipofuscine [18]. La peroxydation de ces segments aggrave encore ce processus en induisant une dysfonction lysosomale bloquant à son tour

${ }^{2}$ La lipofuscine est un complexe de pigments dont le principal est le A2- $\varepsilon$, un chromophore hydrophobe. 
la dégradation des protéines par autophagie [18]. Dans les cellules ARPE-19, une lignée de cellules RPE humaines transformées, l'inhibition de l'autophagie avec la 3-MA (3-methyladenine) induit l'accumulation de lipofuscine. Les altérations lysosomales, l'accumulation de lipofuscine et la réduction de l'autophagie sont donc des événements qui peuvent contribuer au dysfonctionnement des cellules RPE. Ils pourraient influencer l'apparition et la progression de la DMLA.

\section{Le glaucome et les altérations du nerf optique}

Le glaucome regroupe des maladies oculaires caractérisées par des altérations du nerf optique et la perte de vision. Cette pathologie qui affecte 70 millions de personnes à travers le monde, est la deuxième cause de cécité. Elle a pour origine une dégénérescence lente et progressive des cellules ganglionnaires rétiniennes (RGC) et de leurs axones. Le facteur de risque le plus important pour le développement du glaucome est une pression intraoculaire élevée ; la majorité des traitements actuels visent donc à abaisser cette pression intraoculaire. Des dommages au nerf optique surviennent cependant dans certains cas (par exemple le glaucome à tension normale) en dépit de pressions intraoculaires normales [19]. Au cours des dernières années, plusieurs modèles animaux ont été utilisés pour étudier le rôle de l'autophagie dans le glaucome et la mort des RGC. Une étude utilisant un modèle de section du nerf optique chez le rat, a montré une expression accrue de l'ARNm (ARN messager) de plusieurs gènes de l'autophagie, 3 jours après la lésion. La culture de cellules neuronales en l'absence de sérum (déprivation) conduit parallèlement à une augmentation de la mort cellulaire, ce qui suggère un rôle de l'autophagie dans la survie de ces cellules. De ces observations, l'hypothèse que l'autophagie peut constituer une réponse cytoprotectrice dans les RGC, après une lésion axonale, a été émise [20]. Plusieurs études réalisées ultérieurement ont validé cette hypothèse. Dans un modèle murin d'axotomie du nerf optique, l'amélioration pharmacologique de l'autophagie, à l'aide de rapamycine, un inhibiteur de mTOR, a permis de diminuer la dégénérescence des RGC [4] et des animaux n'exprimant pas ATG4B ont présenté une mort cellulaire accrue après axotomie, malgré l'absence de lésions de la rétine en conditions physiologiques [4]. Le traitement à la rapamycine permet également de remédier à la perte de RGC in vivo, dans un modèle d'hypertension oculaire chronique développé chez le rat [21], et in vitro, dans des modèles cellulaires surexprimant la mutation $\varepsilon 50 \mathrm{~K}$ du gène de l'optineurine $\operatorname{OPTN}^{3}[22,23]$. L'effet cytoprotecteur de l'augmentation du flux autophagique a également été démontré dans un modèle d'écrasement du nerf optique qui induit une augmentation du calcium intracellulaire et active la calpaïne, une protéase cytosolique [24]. D'autres études utilisant des inhibiteurs pharmacologiques ne confirment cependant pas totalement ce rôle de protection après lésions axonales $[25,26]$. Néanmoins, ces différentes observations suggèrent un rôle important de l'autophagie dans la survie des RGC en conditions de stress.

\section{Les dystrophies rétiniennes}

Les dystrophies rétiniennes sont un sous-ensemble de maladies dégénératives résultant de la mort des cellules photoréceptrices. Dans certains

\footnotetext{
${ }^{3}$ Mutation associée au glaucome.
}

cas, les bâtonnets sont d'abord affectés, provoquant une perte de vision de nuit. La maladie progresse ensuite et conduit à la mort cellulaire des cônes à l'origine d'une cécité complète [27]. La rétinite pigmentaire (RP), l'une de ces dystrophies, est causée par des mutations qui touchent au moins 35 gènes différents qui se traduisent par des modifications de l'homéostasie des photorécepteurs aboutissant à leur mort cellulaire. Les premières études, utilisant différents modèles de RP, décrivent une activation de l'autophagie, comme en témoigne la lipidation de LC3 (microtubule-associated protein 1 light chain 3), un orthologue d'ATG8 [28, 29]. II n'a cependant pas été déterminé si l'autophagie contribuait activement à la disparition de la cellule, ou si elle constituait une réponse cytoprotectrice [29]. Dans quatre modèles différents de RP, utilisant une analyse par micropuces au moment du pic de mort cellulaire des cônes, Punzo et ses collègues ont montré une surexpression de plusieurs gènes impliqués dans la voie insuline/mTOR, suggérant la possible implication des conditions de déprivation dans les atteintes rétiniennes [30].

La RP autosomique dominante peut avoir pour origine des mutations qui touchent le gène de la rhodopsine. Elle conduit à la mort des cellules photoréceptrices par induction d'un stress du réticulum endoplasmique (RE) faisant suite à l'accumulation de protéines mal conformées [31, 32]. Récemment, une étude a rapporté une amélioration de la fonction visuelle après traitement à la rapamycine chez des souris présentant des mutations affectant le gène codant la rhodopsine [33]. Le traitement à la rapamycine ne semble cependant pas modifier les taux de protéines impliquées dans l'autophagie, les effets bénéfiques de la rapamycine pouvant être liés à l'activation de I'UPR (unfolded protein response) par les protéines mal conformées. Ces données suggèrent que les effets bénéfiques observés pourraient impliquer l'élimination d'agrégats de protéines toxiques, comme cela a été décrit dans d'autres modèles de maladies neurodégénératives.

La souris Rdlo est un modèle de RP humaine largement utilisé. Elle porte une mutation au niveau du gène $P D E 6 B$ (phosphodiesterase $6 B$ ) qui code une phosphodiestérase spécifique des bâtonnets $[34,35]$. Cette mutation provoque une diminution de la fonction de l'enzyme, une augmentation des concentrations de GMPc (guanosine 3',5'-monophosphate cyclique), et la mort cellulaire des bâtonnets [36]. Nous avons récemment décrit, chez ces souris, une accumulation massive de calcium intracellulaire et une activation de la $\mathrm{m}$-calpaïne (calpaïne-2) précédant la mort des cellules photoréceptrices, corrélées à une réduction du flux autophagique [37]. La mort cellulaire des photorécepteurs chez les souris $\mathrm{Rdl0}$ a pu 
être atténuée in vitro, ex vivo et in vivo par des inhibiteurs de calpaïnes et des cathepsines, montrant que le dysfonctionnement lysosomal, qui dépend de la calpaïne, et la diminution d'autophagie étaient reliés à la mort des photorécepteurs [37]. Ces résultats suggèrent que, comme cela a été décrit pour la maladie d'Alzheimer et les accidents vasculaires cérébraux, la mort des cellules photoréceptrices, observée chez les souris $\mathrm{Rdl0}$, peut se produire par un processus impliquant les calpaïnes/ cathepsines qui conduit finalement à des dommages lysosomaux, à la perméabilisation de la membrane lysosomale et à la mort de la cellule [37-39]. Ce processus est souvent accompagné d'une réduction du flux autophagique. II semble donc que les stratégies neuroprotectrices développées pour ces pathologies devraient également inclure une restauration de l'activité lysosomale.

\section{Autophagie et vieillissement de la fonction visuelle}

Le déclin de la fonction visuelle qui accompagne souvent le vieillissement est considéré comme une conséquence de la dégénérescence rétinienne et de la perte de cellules rétiniennes [40]. Le vieillissement est également lié à une diminution générale de l'activité des voies protéolytiques [41, 42] parmi lesquelles la macroautophagie, l'autophagie relayée par les chaperonnes (CMA) et le système ubiquitineprotéasome [43]. Une diminution de l'expression des ARN messagers de plusieurs régulateurs de l'autophagie, corrélée à une baisse du flux autophagique, est observée chez les souris âgées de deux ans [8]. Cet effet est accompagné d'une accumulation de lipofuscine, d'altérations morphologiques des cellules RPE et de la mort des photorécepteurs [8]. De manière surprenante, la CMA est augmentée dans les rétines âgées, ce qui contraste avec la diminution liée à l'âge de l'activité de cette voie décrite dans d'autres organes [44, 45]. À noter que l'on observe également une surexpression de la CMA chez les souris dont le gène ATG5 est supprimé spécifiquement dans les précurseurs neuronaux, soutenant l'idée d'une augmentation généralisée de la CMA lorsque la macroautophagie est altérée, et suggérant que le maintien de l'homéostasie cellulaire dépend du « dialogue » établi entre différentes voies protéolytiques [8]. Élucider le mécanisme à l'origine du vieillissement de la rétine devrait permettre la conception de nouvelles stratégies thérapeutiques afin de prévenir les dysfonctionnements de la fonction visuelle associés au processus normal de vieillissement mais aussi aux pathologies rétiniennes liées à l'âge.

Dans la dernière décennie, un intérêt considérable a été porté sur le développement de nouvelles interventions thérapeutiques ayant pour fondement les processus d'autophagie. Des études sur les effets d'inducteurs de l'autophagie, comme la rapamycine, dans plusieurs modèles expérimentaux de maladies de la rétine et de l'œil, dont le glaucome et la DMLA, ont permis de démontrer leurs effets bénéfiques. À côté du développement d'agents qui stimulent l'autophagie, en augmentant la formation d'autophagosomes, l'amélioration de la fonction lysosomale peut également présenter des avantages certains pour des pathologies ayant pour origine des défauts d'activité lysosomale [46]. Les études que nous avons rapportées mettent en évidence le rôle important de l'autophagie dans le maintien de la fonction rétinienne et des yeux. Des rôles clés joués par l'autophagie dans les cellules photoréceptrices ont été montrés. Ils comprennent: le recyclage des POS (photoreceptor outer segments), la prévention de l'accumulation de lipofuscine et la protection contre l'agrégation des protéines permettant d'empêcher l'oxydation et ainsi de réduire le stress du réticulum endoplasmique. Enfin, dans les RGC, l'autophagie est impliquée dans l'homéostasie axonale. Elle exerce une fonction de protection qui pourrait être induite en diminuant les niveaux de ROS (reactive oxygen species) et en maintenant la fonction mitochondriale. II ressort de la littérature que les altérations de l'autophagie et des voies lysosomales sont impliquées dans de nombreux cas de maladies de l'œil. La baisse de l'activité lysosomale liée à l'âge exacerbe également les défauts d'autophagie. II apparaît donc nécessaire de développer de nouvelles thérapies prenant en compte à la fois les processus d'autophagie et la fonction lysosomale. $\diamond$

\section{SUMMARY}

Autophagy and vision

The retina is a light-sensitive tissue in the vertebrate eye that detects and processes visual images. The eye, including retina, is exposed to a variety of environmental insults and stressors, among which genetic mutations and age-associated alterations that impair their function. Autophagy is a catabolic pathway that promotes the degradation and recycling of cellular components under basal and stress conditions. Proteins, lipids, and even whole organelles are engulfed in autophagosomes and delivered to the lysosome for elimination. Research has only begun to examine the role of autophagy in the visual system. Here, we review the main studies that have sought to explain autophagy's importance for visual function. $\diamond$

\section{LIENS D'INTÉRÊT}

Les auteurs déclarent n'avoir aucun lien d'intérêt concernant les données publiées dans cet article.

\section{RÉFÉRENCES}

1. Boya P, Esteban-Martinez L, Serrano-Puebla A, et al. Autophagy in the eye: development, degeneration, and aging. Prog Retin Eye Res 2016 ; 55 : 206-45.

2. Ichimura $Y$, Komatsu M. Pathophysiological role of autophagy: lesson from autophagy-deficient mouse models. Exp Anim 2011 ; 60 : 329-45.

3. Mariño G, Fernández AF, Cabrera $S$, et al. Autophagy is essential for mouse sense of balance.J Clin Invest $2010 ; 120: 2331-44$.

4. Rodriguez-Muela N, Germain F, Marino G, et al. Autophagy promotes survival of retinal ganglion cells after optic nerve axotomy in mice. Cell Death Differ $2012 ; 19: 162-9$.

5. Rodriguez-Muela N, Boya P. Axonal damage, autophagy and neuronal survival. Autophagy $2012 ; 8: 286-8$.

6. Chen $\mathrm{Y}$, Sawada 0 , Kohno $\mathrm{H}$, et al. Autophagy protects the retina from lightinduced degeneration. J Biol Chem $2013 ; 288$ : 7506-18. 


\section{RÉFÉRENCES}

7. Hara T, Nakamura K, Matsui M, et al. Suppression of basal autophagy in neural cells causes neurodegenerative disease in mice. Nature 2006 ; 441 : 885-9.

8. Rodriguez-Muela N, Koga H, Garcia-Ledo L, et al. Balance between autophagic pathways preserves retinal homeostasis. Aging Cell 2013; 12:478-88.

9. Kim Jy, Zhao H, Martinez J, et al. Noncanonical autophagy promotes the visual cycle. Cell 2013; $154: 365-76$.

10. Perusek L, Sahu B, Parmar T, et al. Di-retinoid-pyridinium-ethanolamine (A2E) accumulation and the maintenance of the visual cycle are independent of Atg7-mediated autophagy in the retinal pigmented epithelium. J Biol Chem 2015 ; 290 : 29035-44.

11. Gan B, Melkoumian ZK, Wu X, et al. Identification of FIP200 interaction with the TSC1-TSC2 complex and its role in regulation of cell size control. J Cell Biol $2005 ; 170$ : 379-89.

12. Yao J, Jia L, Khan N, et al. Deletion of autophagy inducer RBICCl results in degeneration of the retinal pigment epithelium. Autophagy $2015 ; 11$ : 939-53.

13. Zhou Z, Doggett TA, Sene A, et al. Autophagy supports survival and phototransduction protein levels in rod photoreceptors. Cell Death Differ 2015 ; 22 : 488-98.

14. Zhou Z, Vinberg F, Schottler F, et al. Autophagy supports color vision. Autophagy 2015 ; 11 : 1821-32.

15. Isenmann S, Kretz A, Cellerino A. Molecular determinants of retinal ganglion cell development, survival, and regeneration. Prog Retin Eye Res $2003 ; 22: 483-543$.

16. Kurz T, Karlsson M, Brunk UT, et al. ARPE-19 retinal pigment epithelial cells are highly resistant to oxidative stress and exercise strict control over their lysosomal redox-active iron. Autophagy $2009 ; 5: 494-501$.

17. Bergmann M, Schutt F, Holz FG, Kopitz J. Inhibition of the ATP-driven proton pump in RPE lysosomes by the major lipofuscin fluorophore A2- $\varepsilon$ may contribute to the pathogenesis of agerelated macular degeneration. Faseb J $2004 ; 18: 562-4$

18. Krohne TU, Stratmann NK, Kopitz J, Holz FG. Effects of lipid peroxidation products on lipofuscinogenesis and autophagy in human retinal pigment epithelial cells. Exp Eye Res 2010 $90: 465-71$.

19. Qu J, Wang D, Grosskreutz CL. Mechanisms of retinal ganglion cell injury and defense in glaucoma. Exp Eye Res $2010 ; 91: 48-53$

20. Kim SH, Munemasa Y, Kwong JM, et al. Activation of autophagy in retinal ganglion cells. J Neurosci Res $2008 ; 86: 2943-51$

21. Su W, Li Z, Jia Y, Zhuo Y. Rapamycin is neuroprotective in a rat chronic hypertensive glaucoma model. PLoS One 2014 ; 9 : e99719.

22. Chalasani ML, Kumari A, Radha V, Swarup G. \&50K-OPTN-induced retinal cell death involves the Rab GTPase-activating protein, TBC1D17 mediated block in autophagy. PLoS One 2014 ; 9 : e95758.

23. Ying $\mathrm{H}$, Turturro $\mathrm{S}$, Nguyen $\mathrm{T}$, et al. Induction of autophagy in rats upon overexpression of wildtype and mutant optineurin gene. BMC Cell Biol $2015 ; 16: 14$

24. Ribas VT, Koch JC, Michel U, et al. Attenuation of axonal degeneration by calcium channe inhibitors improves retinal ganglion cell survival and regeneration after optic nerve crush. Mol Neurobiol 2017 ; 54 : 72-86.

25. Knoferle J, Koch JC, Ostendorf T, et al. Mechanisms of acute axonal degeneration in the optic nerve in vivo. Proc Natl Acad Sci USA $2010 ; 107: 6064-9$.

26. Park HY, Kim JH, Park CK. Activation of autophagy induces retinal ganglion cell death in a chronic hypertensive glaucoma model. Cell Death Dis $2012 ; 3$ : e290.

27. Lev S. Molecular aspects of retinal degenerative diseases. Cell Mol Neurobiol $2001 ; 21$ : 575-89.

28. Lohr HR, Kuntchithapautham K, Sharma AK, Rohrer B. Multiple, parallel cellular suicide mechanisms participate in photoreceptor cell death. Exp Eye Res $2006 ; 83: 380-9$.

29. Kunchithapautham K, Rohrer B. Autophagy is one of the multiple mechanisms active in photoreceptor degeneration. Autophagy $2007 ; 3: 65-6$.
30. Punzo C, Kornacker K, Cepko CL. Stimulation of the insulin/mTOR pathway delays cone death in a mouse model of retinitis pigmentosa. Nat Neurosci $2009 ; 12: 44-52$

31. Athanasiou D, Aguila M, Bevilacqua D, et al. The cell stress machinery and retinal degeneration. FEBS Lett $2013 ; 587: 2008-17$.

32. Kroeger H, LaVail MM, Lin JH. Endoplasmic reticulum stress in vertebrate mutant rhodopsin models of retinal degeneration. Adv Exp Med Biol 2014 ; $801: 585-92$

33. Sizova OS, Shinde VM, Lenox AR, Gorbatyuk MS. Modulation of cellular signaling pathways in P23H rhodopsin photoreceptors. Cell Signal $2014 ; 26$ : $665-72$.

34. Gargini C, Terzibasi $\varepsilon$, Mazzoni F, Strettoi $\varepsilon$. Retinal organization in the retinal degeneration 10 ( $\mathrm{rdl}$ ) mutant mouse: a morphological and ERG study. Comp Neurol 2007 ; $500: 222-38$.

35. Barhoum R, Martinez-Navarrete G, Corrochano S, et al. Functional and structural modifications during retinal degeneration in the $\mathrm{rdl} 10$ mouse. Neuroscience $2008 ; 155: 698-713$.

36. Corrochano S, Barhoum R, Boya P, et al. Attenuation of vision loss and delay in apoptosis of photoreceptors induced by proinsulin in a mouse model of retinitis pigmentosa. Invest Ophthalmol Vis Sci 2008 ; 49 : 4188-94.

37. Rodriguez-Muela N, Hernandez-Pinto AM, Serrano-Puebla A, et al. Lysosomal membrane permeabilization and autophagy blockade contribute to photoreceptor cell death in a mouse model of retinitis pigmentosa. Cell Death Differ $2015 ; 22$ : 476-87.

38. Yamashima T. Reconsider Alzheimer's disease by the 'calpain-cathepsin hypothesis': a perspective review. Prog Neurobiol 2013 ; 105 : 1-23.

39. Serrano-Puebla A, Boya P. Lysosomal membrane permeabilization in cell death: new evidence and implications for health and disease. Ann Ny Acad Sci 2016 ; $1371: 30-44$.

40. Militante J, Lombardini JB. Age-related retinal degeneration in anima models of aging: possible involvement of taurine deficiency and oxidative stress. Neurochem Res $2004 ; 29: 151-60$.

41. Rubinsztein DC, Marino G, Kroemer G. Autophagy and aging. Cell 2011 ; 146 682-95.

42. Kroemer $\mathrm{G}$. Autophagy: a druggable process that is deregulated in aging and human disease. J Clin Invest $2015 ; 125: 1-4$

43. Martinez-Lopez N, Athonvarangkul D, Singh R. Autophagy and aging. Adv Exp Med Biol $2015 ; 847: 73-87$

44. Cuervo AM, Dice JF. Age-related decline in chaperone-mediated autophagy. J Biol Chem $2000 ; 275: 31505-13$.

45. Zhang C, Cuervo AM. Restoration of chaperone-mediated autophagy in aging liver improves cellular maintenance and hepatic function. Nat Med $2008 ; 14: 959-65$

46. Kim YC, Guan KL. mTOR: a pharmacologic target for autophagy regulation. J Clin Invest $2015 ; 125: 25-32$.

47. Morel $\varepsilon$. La formation de l'autophagosome : un nouveau défi pour le biologiste cellulaire. Med Sci (Paris) 2017 ; $33: 217-20$.

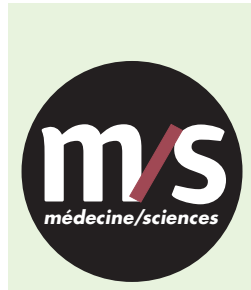

$>$ Grâce à $m / s$, vivez en direct les progrès

Tarifs d'abonnement m/s - 2017 des sciences biologiques et médicales

Abonnez-vous

à médecine/sciences

Bulletin d'abonnement

page 362 dans ce numéro de $\mathrm{m} / \mathrm{s}$

\section{TIRÉS À PART}

P. Boya
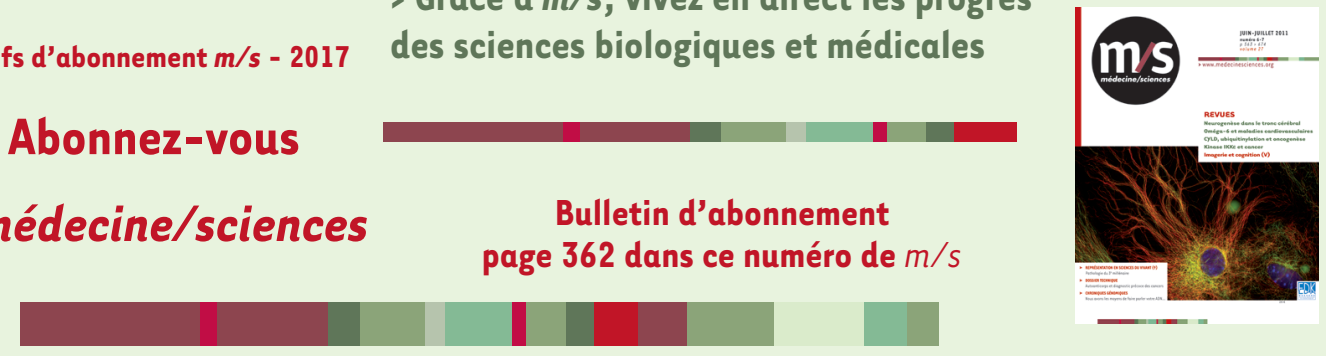